\title{
Immune-System Inspired Approach for Decentralized Multi-Agent Control
}

\author{
S. German \\ School of Aerospace, \\ Transport and Manufacturing, \\ Cranfield University, Bedford, \\ MK430AL, UK \\ Email: servando.german.serrano@gmail.com
}

\author{
H.-S. Shin \\ School of Aerospace, \\ Transport and Manufacturing, \\ Cranfield University, Bedford, \\ MK430AL, UK \\ Email: h.shin@cranfield.ac.uk
}

\author{
A. Tsourdos \\ School of Aerospace, \\ Transport and Manufacturing, \\ Cranfield University, Bedford, \\ MK430AL, UK \\ Email: a.tsourdos@cranfield.ac.uk
}

\begin{abstract}
This paper contains the first steps towards the development of a fully decentralized system framework. The novel approach that has been taken is derived from the inherent properties of the immune system. An assessment of the proposed control architecture has been performed by comparison with a more typical approach under a search and suppress kind of mission for an unmanned fleet.
\end{abstract}

Index Terms-immune system, decentralized architecture

\section{INTRODUCTION}

During the last years, the main objective of the technology involved in Unmanned Systems, has been focused in the development of multitasking platforms, which are capable of performing different kinds of missions without making significant changes on them. This approach leads to very capable fleets, able to succeed in a broad range of missions but, extremely weak against certain kinds of attacks that can can be exploited by the adversaries to completely annihilate all fleet members.

Centralized control architectures are typically employed in these fleets, to achieve the required cooperative behaviors to successfully complete their mission. This solution works fairly good for small fleets, but the expensive computational and communication requirements prevents this control implementation from being scalable as the fleet increases in size; being it also quite vulnerable to the loss of its central controller.

The aforementioned facts are the main reasons behind the work that has been conducted as

- There is a need of exploring decentralized architectures that would provide the unmanned fleet with the required flexibility to achieve complex tasks.

- New fleet configurations are required, based on different platforms, specialized in a particular set of tasks; leading to a reduction in the required fleet budget, but providing the fleet with the arising complex behaviours that it needs to succeed.

The work conducted during this research project aims to:

- Develop a highly innovative decentralized control system framework, for multi-agent fleet cooperation in an unknown and dynamic environment.
- Proposal of a new fleet configuration, using an heterogeneous approach regarding each fleet member capabilities, as contrast with the current trends with homogeneous configuration.

The following paper is organized as follows, in section II the basis of decentralized multi-agent systems are mentioned Section III summarizes the main characteristics of the Immune system along with a description of different computational models associated to them. Following, the commonalities that can be found between the immune system behaviours and those that can be found on-board an unmanned vehicle are presented in section IV; in section V the developed framework model and implementation is explained. Section VI contains the chosen simulation environment parameters and characteristics to test the proposed framework. Next, in section VII the simulation results are analyzed and, finally, the reached conclusions are presented in section VIII.

\section{Decentralized MUltiagent Systems}

In a self-organizing multi-agent system, the mutual interaction of its agents' behaviors generates, as a side effect, a group behavior; while the dynamic environment works as a driving force to organize the group behavior, as cited in [1] and [2].

A successful cooperative operation algorithm reduces cost and enhances reconfigurability and robustness; but the design is challenging due to the increase of complexity and nonlinearity of the overall system as the number of agents increase.

Different approaches have been examined and used as an attempt to control and organize a multi-agent system, each one with different associated problems:

- Centralized operation, which carries large computation and communication requirements with it.

- Behavioral artificial intelligence methods, these implementations have bottlenecks when coping with complex and dynamic environments.

- New techniques based on macroscopic primitives, such as PSO algorithms, that are inspired by the emergent behaviours that can be observed in bird flocks or fish schools. 
However, these methods are in a theoretical stage or working on function peak value problems, with only a few being used in practical engineering problems.

This paper presents a novel technique, where the collective behavior emerges independently of any centralized control as the system distributes its overall functionality among the smaller, less expensive and cooperative agents, where each agent bases its own actions on its available and local knowledge of the surrounding environment.

\section{THE IMMUNE SYSTEM AS AN APPROPRIATE APPROACH}

The following properties, cited in [3], make the immune system the perfect source of inspiration to develop a fully decentralized architecture:

- Distributed and self organized: the highly complex behavior of the immune system arises as a result of the individual behaviors of billions of agents distributed throughout, with no central controller; where each cell reacts with regard to its local knowledge of the environment. The organized response emerges as a system-wide property that is derived from the low-level agent's behavior.

- Learning, adaptation and memory capabilities: it is able to recognize previously unseen pathogens, being able to recognize them in posterior encounters to cope with them in a faster and more efficient way. This fact implies that the immune system possesses a sort of 'memory' and, hence, the capability of 'learning'.

- Pattern recognition: its is capable recognizing a wide range of pathogens as a whole, while each agent can only recognize a limited range of pathogens through their own receptors.

- Classification: the immune system is capable of distinguishing between harmful and non-harmful substances, doing so by just accessing information about its own molecules.

Because of these inherent properties, the immune system is a highly intelligent cooperative system that self-organizes to mount a proper and adequate response to invading antigens.

Based on these observations of the immune system, in the swarm intelligence algorithm approach, each agent perceives its environment and its neighbors' behaviors, with local peer to peer communications, determining then its own behavior independently; in order to cooperate with other agents towards the common goal.

\section{A. Brief description of the Immune System}

The immune system is composed of two distinguished parts, the innate immune system, which is formed of static defenses, such as skin; and the adaptive immune system, which is responsible for the generation of specific reactions to cope with infectious agents. The proposed decentralized framework is inspired by the characteristics of the latter part, the adaptive immune system.

The adaptive immune system is responsible for the production of a specific reaction that copes with the infectious agents that have surpassed the innate immune system. The basic components of the adaptive immune system are white blood cells, known as lymphocytes; of which there are two main kinds, B-cells and T-celles.

A deeper description on the fundamentals of the immune system, than the one presented in this paper can be found in [2] and [4].

\section{B. Computational models based on the Immune System}

The main computational models that have been developed and implemented are:

- Bone marrow models.

- Clonal selection.

- Negative-selection theorem.

- Danger theory.

- Immunized computational systems (ICS).

- Idiotypic network model.

The usability of one model or another is subjected to the available knowledge of the considered problem. For the purpose of this paper, the considered problem is the development of a completely decentralized multi-task allocation algorithm, in order to generate complex group behaviors by making use of the limited and simpler behavior of each unmanned vehicle of a fleet. Hence, the main aspects of the immune system that act as inspiration for the novel algorithm are Clonal Selection and Idiotypic Network Model.

1) Clonal Selection: Clonal selection based algorithms try to capture the mechanisms of B-cell proliferation, driven only by the presence of antigens, improving this way the B-cells binding affinity. Using a process known as affinity maturation, the receptors of the B-cell are mutated, the subsequent B-cell selection results in a population that is better adapted to the present antigen than its ancestor population. These algorithms capture the properties of learning, memory, adaptation and pattern recognition of the immune system.

When an antigen enters a living body, not all of the available antibodies that come into contact with it will be able to recognize said antigen; some of them have higher affinity and are, thus, more suited to cope with it in order to make it disappear. Those B-cells, whose antibodies have higher affinity to the encountered antigen, are induced to replicate further more than those with smaller affinity.

As a result, the population of B-cells evolves towards the extermination of the antigen in a short period of time. Once the antigen has been erased, all the B-cells recover their initial proliferation rate, so if the antigen appears again is easier for the system cope with it.

Translating this to the computational environment; first a set of antigens is input to the algorithm, with a set of memory B-cells as output. At the first iteration of the algorithm a set of randomly initialized B-cells is chosen, being cloned afterwards proportionally to their affinity to the presented antigens. The higher affinity cells replace those of lower affinity from the previous generation. Following, each generation of B-cells is mutated at a rate inversely proportional to their affinity, allowing this way for the lower affinity cells to develop to cope with the antigens. During the last stages of the algorithm, 
those cells with the highest affinity compete for a place in the set of memory cells.

It is possible to tailor this algorithm approach for optimization problems by removing the set of antigens and evaluating directly the optimization function as the affinity function of the B-cells.

2) Idiotypic Network Model: The Idiotypic Network Model was proposed by Jerne, and its basic idea is that the immune system is a regulated network of molecules and cells which are capable of recognizing each other. As antibody molecules are created by random genetic mechanisms, they must look like novel molecules to the rest of the immune system, and must therefore be treated like antigens. Since antibodies have epitopes, they are capable of being recognized by other antibodies and, a network of B-cells occurs due to the matching of the paratopes against the idiotopes on other B-cells, where the same antibody could interact with more than one antibody. The immune system will recognize its own antibodies as if they were foreign and will make antibodies against them.

An antibody is stimulated when it recognizes other types, and suppressed if it is recognized. There exists a balance which is established between recognizing and being recognized that produces a network of coupled stimulation-suppression phenomenon that maintains a stable equilibrium of good antibodies for future use. As the antibody matures, it recognizes the antigen with a higher degree of accuracy and, once the antigen is removed, the antibody network helps in keeping the immune system from extinguishing itself. A stable population is maintained as 'memory' that will be useful for future encounter with a similar antigen.

In the idiotypic network, the immune system operates at a steady state in absence of antigen; an antigen simply causes a perturbation away from this steady state. Upon the termination of the response to the antigen, the system would return to a steady state, either the previous one or a new one.

This theory does not require the presence of antigens to stimulate an immune response. The dynamics are governed by both the presence of antigens and antibodies. The increase or decrease of the concentration of a set of lymphocyte clones and the responding B-cells can simulated using a non-linear differential equation, [1], [2] and [4].

The mathematical model of this model is provided in section $\mathrm{V}$, where the full modeling and implementation of the idiotypic network is devised.

\section{Analogy Between Immune System and UAS BEHAVIORS}

In order to make use of the Immune System characteristics as the starting point for the development of a fully decentralized system, it is necessary to make an analogy between the Unmanned Fleet and the agents of the Immune System.

Considering first the different unmanned vehicles that compose a general fleet, each one of them would have different set of possible simple behaviors, or tasks, that each can accomplish as a result of their different on-board sensors or platform characteristics. Thus, it is feasible to consider each vehicle as a B-cell with different antibodies, which are equivalent to the different behaviors. In the same sense, the different tasks or threats that appear in the dynamic environment the fleet has to perform can be regarded as the antigens that the immune system has to cope with.

In this way, each platform within the fleet would be capable of coping with certain tasks or threats depending on its capabilities, hence the need to generate a cooperative behavior to accomplish the fleet tasks.

\section{Modeling And Implementation of the IDIOTyPiC NETWORK}

The mathematical model that represents B-cell molecules' population dynamics, was proposed by Farmer [5],

$$
\begin{aligned}
\dot{x}_{i}= & k_{3}\left[\sum_{j=1}^{n} S_{i j} x_{i} y_{j}+\sum_{j=1}^{N} M_{j i} x_{i} x_{j}-k_{1} \sum_{j=1}^{N} N_{j i} x_{i} x_{j}\right] \\
& -k_{2} x_{i} \\
= & k_{3}\left[T_{1}+T_{2}-k_{1} T_{3}\right]-k_{2} T_{4}
\end{aligned}
$$

Farmer's equation, 1, represents how the concentration of antibody, $x_{i}$, evolves, with regard to the presence of antigens, $y_{j}$, enclosed in term $T_{1}$, stimulation and suppression due to other antibodies, $x_{j}$, represented by terms $T_{2}$ and $T_{3}$, respectively. And, natural death rate of antibodies, which is expressed by term $T_{4}$. Where $N$ is the number of different antibodies, $n$ is the number of antigens and constant $k_{1}$, represents asymmetry between stimulation and suppression, $k_{2}$ and $k_{3}$ are weighting factors of natural death rate and the result of stimulation and suppression due to the idiotypic network, respectively.

Regarding the scope of this paper, the last term of the equation, $T_{4}$ is not considered, as the different behaviors on each Unmanned Vehicle are not considered to die during its mission.

In [6], the implementation of a full artificial immune system is carried out, following equation 1 , for a single robot; this work has been used as the starting point for the development of the novel decentralized framework proposed in this paper.

Next the required steps for the implementation are outlined.

1) Preparation of the Idiotope matrix The Idiotope matrix represents which combinations of antigen and antibody are considered harmful in some sense for the unmanned vehicle. Taking this sense and, considering each row of the matrix for each behavior and each column related to each antigen; the matrix is initialized. Using previous knowledge of pairs of antibody-antigen that are supposed to be harmful, a positive value is assigned to the corresponding position within the idiotope matrix, if certain pair is a prioriunknown to be dangerous for the vehicle or not, a value of zero is assigned to the corresponding position in the matrix. In order to initialize the matrix at first, it is considered that the sum across each row, i.e. for each behavior, has to be equal to 1 , or 0 if the behavior is not taken as harmful, and; a practical way to allocate 
the values in the matrix, if no previous knowledge of how harmful one of the vehicle's behavior regarding the different antigens is, is to take an average among those behaviors that are seen as detrimental for the vehicle's operation.

2) Initialization of the Paratope matrix The paratope matrix shows affinity between the different behaviors and antigens, indicating which behavior is more suitable to tackle the encountered antigen.

A random initialization is proposed, in order for each agent within the unmanned fleet to learn as they are developing their mission or tasks.

Following the work in 4, the paratope matrix can be initialized as follows. Considering first that no negatives affinities are possible, hence, a behavior that is not suitable for a certain task will have a value of 0 allocated in the corresponding position of the paratope matrix; the matrix is initialized with numbers ranging from 0.5 to 0.75 , modifying them so that the average value across each row, i.e. for each behavior, is equal to 0.625 .

3) Antibody concentrations Regarding antibody (behavior) concentrations, they are initialized with a value of 1 and total concentration on-board each fleet agent is kept constant during its whole mission. So, after each time Farmer's equation is used and the concentration of each behavior is updated, they are all normalized so the sum of the concentrations of all the behaviors remain the same.

4) Development of Farmer's equation After setting the initial idiotope and paratope matrices and the antibody concentrations for each unmanned vehicle, it is possible to implement Farmer's equation in order to simulate the evolution of antibody concentration so the vehicle is able to select its most appropriated behavior with regard to the local knowledge of the world available to it.

\section{5) Learning scheme}

In order for the fleet to adapt and evolve as its mission progresses, it is necessary for each unmanned agent, that composes the system, to evaluate its chosen behavior, after coping with an encountered antigen, with regard to certain metric that will depend on the unmanned fleet and its mission; leading to an increase in the behavior's associated paratope matrix value if the metric evaluation is successful or to a decrease in said value if the opposite happens.

6) Idiotypic Network algorithm for a whole fleet

Taking now the description on how to implement a full artificial immune system for each vehicle, it can be easily expanded for the whole fleet as explained next.

The fleet's mission starts with each vehicle performing an initial behavior up until one of them encounters an antigen. Once, an antigen has been encountered by a certain unmanned vehicle, it selects its most appropriate behavior as a result of the different stimulations and suppressions of antibodies as aforementioned.

Next, it communicates to those vehicles within its communication range that an antigen has been found by sending vector $G$, which encloses the priority order if more than one antigen is encountered as the same time. If any of those unmanned vehicles that have received vector $G$ is already taking care of an antigen, it will disregard the communication until it has finished performing its current task.

Apart from vector $G$, each vehicle, that is able to go cope with the encountered antigen, will communicate its current idiotope and paratope matrices to those others that are available to cope with the encountered threat.

As each vehicle receives the others' idiotope and paratope matrices, they mount bigger ones by stacking those that each has received one after another

Following, each vehicle computes the Idiotypic Network, by making use of the new matrices that has built: first calculating the antigenic antibody using $P_{\text {global }}$, as its paratope matrix, to obtain next, the induced stimulations and suppressions. After calculating $T_{g}$, the behavior that has greater value from the first piece of $T_{g}$, which corresponds to those behavior available for the vehicle is selected as the suitable behavior for the considered antigen situation.

Allowing communications for a predefined period of time results in each vehicle within the fleet to receive information regarding the encountered set of antigens and the other vehicles current immune system matrices, generating a network that makes use of the local knowledge available to each vehicle to organize itself.

More than one vehicle can select the same behavior and try to cope with the encountered antigen at the same time. A binding affinity function can be defined, that takes into account distance to the antigen, maximum speed of the vehicle and remaining endurance among others; so, between those vehicles that select the same behavior to cope with the antigen, it is possible for them to decide which one or ones go cope with the antigen.

\section{UNMANNED FLEET SIMULATION}

In order to assess the suitability and performance of the proposed decentralized control algorithm a Search and Suppress mission-type simulation has been conducted, with two different Unmanned Combat Aerial Vehicle fleet configurations.

The proposed mission requires the fleet to search a $20 \times$ $20 \mathrm{~km}$ square, with unknown threats, in order to find the main objective that has to be destroyed. The environment is set with SAM sites and enemy UAVs that patrol the environment, hence the fleet has to cope with these threats as they are being found until the main objective is found. A possible configuration for the environment can be seen in 1 .

Figure 1 shows with a star the main objective, with circles the considered range of the SAM sites and the blue eightshaped lines represent the enemy UAVs flight path as they patrol around the main objective of the mission.

Considering now the UCAV fleets, they are the same regarding the aerial platform, but different regarding the capabilities on-board each vehicle. One fleet is composed of multi-tasking 


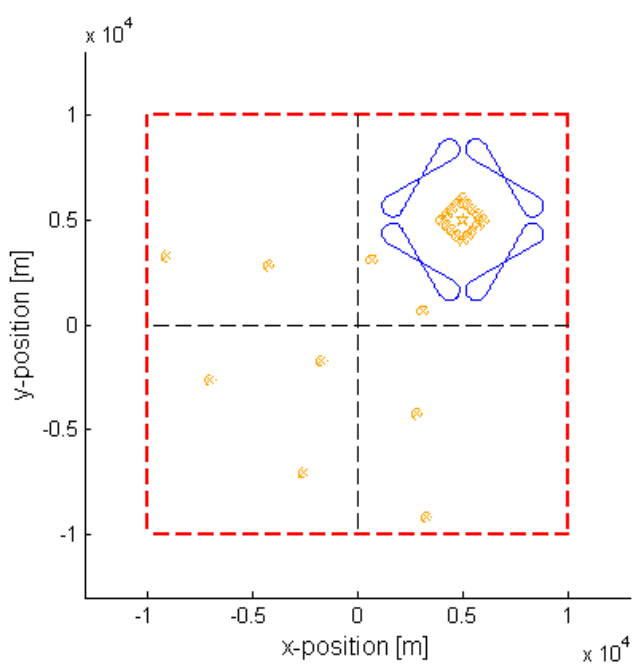

Fig. 1: Enemy environment.

platforms, hence being homogeneously build; and the other is heterogeneous, since different capabilities are set on each vehicle.

Each multi-task vehicle is capable of searching and coping with ground and aerial enemy targets; whereas for the heterogeneous fleet the vehicles that compose it are divided into three different groups, depending on the capabilities of each platform, search specialized, ground target specialized and aerial target specialized ones.

Since a $2 D$ simulation has been performed as the first step to assess the complex and cooperative emergent behaviors reachable by the novel decentralized architecture proposed in this paper, some simplifications have been taken into account:

- The SAM sites' detection range has been reduced when compared with real ones as no complex maneuvers are achievable in a $2 D$ environment.

- Different hitting probabilities are taken into account in order to assess whether a fleet member of an encountered threat is destroyed, when they meet.

- Considering the same budget for each vehicle within the fleet, it is feasible to assume that the agents among the heterogeneous fleet would have enhanced capabilities when compared to those on the homogeneous fleet, as the budget for each vehicle within the heterogeneous fleet is employed to provide them with the required capabilities for one specific task.

- Each agent within the homogeneous fleet has the following set of behaviors:

- Search.

- Attack SAM site.

- Attack UAV.

- Refuel.

- Each search specialized vehicle within the heterogeneous fleet is capable of:

- Search.

- Communicate ground target found.
- Communicate UAV found.

- Refuel.

- The ground target specialized agents can:

- Loiter.

- Attack ground target.

- Avoid enemy UAV.

- Refuel.

- The UAV target specialized UCAVs can:

- Loiter.

- Attack UAV.

- Avoid SAM site.

- Refuel.

Regarding mission development for each fleet, different approaches, taking into account the differences between both fleet configurations are made: homogeneous fleet and heterogeneous fleet.

\section{SiMULATION RESULTS}

A Monte Carlo simulation has been conducted for both fleets in order to evaluate each fleet performance, taking first the percentage of missions that are successfully completed, i.e. the main objective is found and destroyed; followed by an evaluation of the time taken to complete said mission and the number of fleet members that survive the mission.

Different fleet sizes have been considered, so it would be possible to corroborate the clear idea that as the fleet size increases the number of completed missions increases as well.

Figure 2 shows the percentage of successful missions accomplished by both fleets; although the trend is the same as the fleet size increases; the heterogeneous fleet is able to complete the mission a higher percentage of the times.



Fig. 2: Percentage of successful missions.

The number of remaining fleet members and the time taken for the fleet to complete the mission are displayed in figures 3 and 4 respectively. Where the mean value and the standard deviation are shown.

It is obvious the enhancement that the immune system implementation and the heterogeneous distribution of the fleet can achieve, as the generated complex emergent behaviors and the different capabilities on-board each UCAV make the fleet 


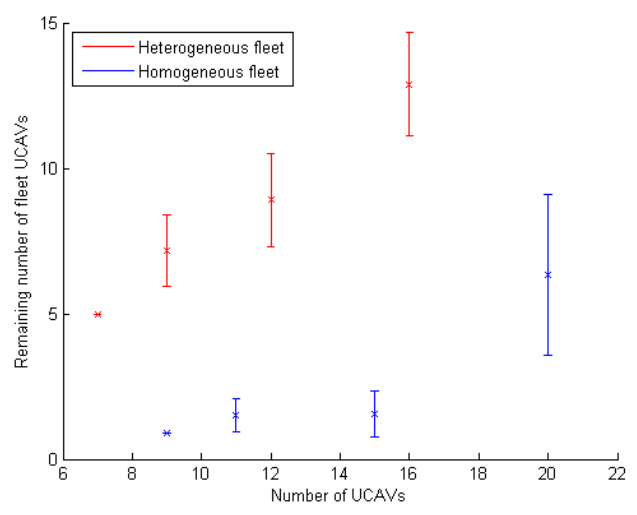

Fig. 3: Remaining fleet members.

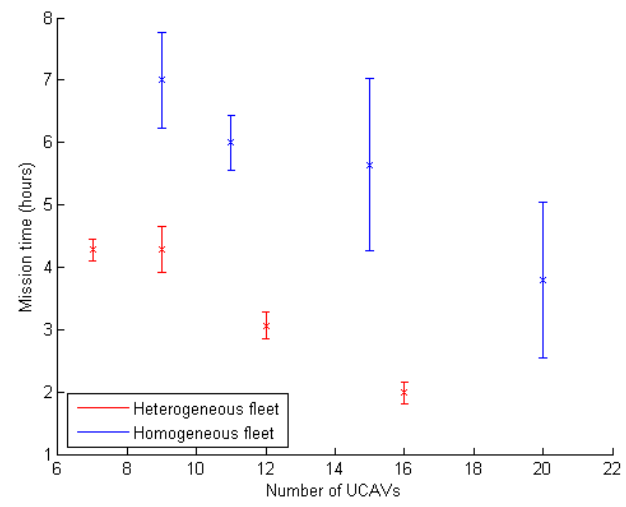

Fig. 4: Mission times.

capable to adapt to the unknown and changing environment, making it much more resilient to enemy attacks and environmental threats that the typical homogeneous configuration.

\section{CONCLUSIONS}

The main aim of this research has been focused in the development of a fully decentralized control system for a fleet of unmanned vehicles, aiming to achieve the flexibility, robustness and fault tolerance requirements that a system like that is required to have.

The immune system has been chosen as source of inspiration due to its inherent flexibility regarding the different behaviors and antigens that it can cope with, as it is not limited, since it is possible to keep adding behaviors and antigens, meaning new different platforms and threats or situations to cope with; without having to make nothing but slight changes in how the system works.

The robust capabilities that a distributed system needs to have, regarding failure or destruction of some of the fleet members; have been assessed through the Monte Carlo simulation leading to the conclusion that the developed algorithm can be highly scalable for greater fleet sizes as each vehicle acts with regard to its available local knowledge of the environment, while influenced by those neighbors within its communication range. As well, as the limited communication and computational requirements that are needed on-board each vehicle to obtain the global idiotope and paratope matrices to select its most appropriate behavior, contribute to the algorithm scalability.

Even though the simulation has been carried out choosing a fleet composed of the same aerial platform but with different capabilities, the proposed framework opens the door to the employment of more complex fleets. Being possible to combine different sorts of platforms, ranging from aerial to ground ones, and even groups of people, where their different actions can be considered as antigens that may trigger new behaviors on the unmanned vehicles. Hence, more complex collaborative group behaviors can be achieved, which can be really useful in other kind of mission, such as Search and Rescue or Surveillance ones.

A new approach to fleet configurations is proposed as well, where the fleet would be composed heterogeneously, opposed to the current trends of having more expensive multi-task platforms.

The results shown by the Monte Carlo simulation drive to the conclusion that the usage of an heterogeneous fleet, instead of the common homogeneous ones, can enhance the performance without increasing the fleet size, but combining different sorts of platforms. Making it possible to generate complex group behaviors that would be seen as unexpected for the enemy, decreasing this way the fleet's weakness; whereas with homogeneous fleets where all the agents behave in the same way, being predictable, the heterogeneous fleet is not.

The proposal of an heterogeneous fleet, can lead to a decrease in total fleet cost, as cheaper unmanned vehicles can be used as first in order to unveil the possible threats that the environment hides; in order to keep those that are more expensive due to its payload in a safe place until they are strictly needed to cope with those tasks that the cheaper ones are not able to carry out.

\section{REFERENCES}

[1] N. Mitsumoto, T. Fukuda, F. Arai, H. Tadashi, and T. Idogaki. Selforganizing multiple robotic system (a population control through biologically inspired immune network architecture). In Proceedings - IEEE International Conference on Robotics and Automation, volume 2, pages 1614-1619, 1996.

[2] M. Xia L. Weng, Q. Liu and Y.D. Song. Immune network-based swarm intelligence and its application to unmanned aerial vehicle (uav) swarm coordination. Neurocomputing, 125:134-141, 2014.

[3] P. S. Andrews M. Read and J. Timmis. An introduction to artificial immune systems. In Handbook of Natural Computing. Springer, Berlin, 2012.

[4] K. Krishna Kumar. Artificial immune system approaches for aerospace applications. 41st Aerospace Sciences Meeting and Exhibit, 2003.

[5] J Doyne Farmer, Norman H Packard, and Alan S Perelson. The immune system, adaptation, and machine learning. Physica D: Nonlinear Phenomena, 22(1):187-204, 1986.

[6] A. M. Whitbrook, U. Aickelin, and J. M. Garibaldi. Idiotypic immune networks in mobile-robot control. IEEE Transactions on Systems, Man, and Cybernetics, Part B: Cybernetics, 37(6):1581-1598, 2007. 
2016-08-08

\section{Immune-system inspired approach for decentralized multi-agent control}

German, S.

Institute of Electrical and Electronics Engineers

S. German, H.-S. Shin and A. Tsourdos. Immune-system inspired approach for decentralized multi-agent control. 24th Mediterranean Conference on Control and Automation, 21-24 June 2016, Athens, Greece.

10.1109/MED.2016.7536048

Downloaded from Cranfield Library Services E-Repository 